\title{
Further evidence of long-term thermospheric density change using a new method of satellite ballistic coefficient estimation
}

\author{
Arrun Saunders, ${ }^{1}$ Hugh Lewis, ${ }^{1}$ and Graham Swinerd ${ }^{1}$ \\ Received 8 December 2010; revised 8 July 2011; accepted 20 July 2011; published 13 October 2011. \\ [1] Building on work from previous studies a strong case is presented for the existence of \\ a long-term density decline in the thermosphere. Using a specially developed orbital \\ propagator to predict satellite orbit evolution, combined with a new and accurate method \\ of determining satellite ballistic coefficients, a long-term thermospheric density change \\ has been detected using a different method compared to previous studies. Over a 40-year \\ period between the years 1970 and 2010, thermospheric density has appeared to reduce by \\ a few percent per decade. However, the results do not show the thermospheric density \\ reduction to vary linearly with time. Therefore, by analyzing the derived density data over \\ varying solar activity levels, as well as performing a Fourier spectral analysis to highlight \\ any periodicities, connections with physical phenomena, where possible, are proposed.
}

Citation: Saunders, A., H. Lewis, and G. Swinerd (2011), Further evidence of long-term thermospheric density change using a new method of satellite ballistic coefficient estimation, J. Geophys. Res., 116, A00H10, doi:10.1029/2010JA016358.

\section{Introduction}

[2] As the space environment grows increasingly populated with newly launched objects, mechanisms affecting satellite and space debris orbits are of great relevance in the process of orbit determination. One of the factors that affects objects in low Earth orbit (LEO) is aerodynamic drag caused by the object's movement through the Earth's atmosphere. As the drag encountered by satellites depends on the local mass density of the atmosphere, knowledge of long-term trends in atmospheric density are extremely valuable in predictions of the lifetimes of objects in LEO. Therefore, knowing atmospheric density trends has applications in predicting the future space environment, as well as being a tool for satellite operators; and thus providing the driving forces for this study.

[3] Initial work regarding upper atmospheric density change focused on the theoretical effect of doubling the concentrations of $\mathrm{CO}_{2}$ and $\mathrm{CH}_{4}$ [Roble and Dickinson, 1989]. The results predicted a temperature reduction of $10 \mathrm{~K}$ in the thermosphere, increasing to $50 \mathrm{~K}$ at the lower altitudes of the mesosphere. In a later study [Roble and Ramesh, 2002], the atmospheric constituents that were predicted to affect the thermosphere most significantly were the greenhouse gases, $\mathrm{CO}_{2}$ and NO. The mechanism to reduce thermospheric densities was due to energetic (hot) oxygen atoms colliding with the greenhouse gas molecules. They in turn transfer some of their energy that is then emitted into space as infrared radiation; resulting in a net cooling effect. As the atmospheric gas molecules then, in general, have less

\footnotetext{
${ }^{1}$ Astronautics Research Group, School of Engineering Sciences, University of Southampton, Highfield, UK.

Copyright 2011 by the American Geophysical Union. 0148-0227/11/2010JA016358
}

energy, their trajectories do not reach as high an altitude and therefore local density decreases.

[4] During the last decade there have been several empirical studies into the existence of a long-term density decline in the thermosphere: The first of these [Keating et al., 2000] used the long-term orbital decay of five satellites with perigee altitudes averaging around $350 \mathrm{~km}$. The results provide evidence of a declining average of $9.8 \pm 2.5 \%$ in thermospheric densities over a 20-year period between 1976 and 1996. This method incorporated an analytical approach [King-Hele, 1987] using the orbital elements given explicitly by the freely available two-line element (TLE) sets. However, using orbital elements directly from the TLE sets gives inaccurate results due to the averaging process used to create the TLE sets. This will be discussed in more detail in a later section. Therefore, to increase the scope of such investigations, more sophisticated methods were required.

[5] Using a new technique to derive thermospheric mass density from TLE sets [Picone et al., 2005], a more detailed study was performed using 27 long-lived near-Earth orbiting objects [Emmert et al., 2004]. Their satellite data covered all levels of solar variability spanning the time period of 1966 2001. The results concluded a density trend of $-2 \%$ per decade at $200 \mathrm{~km}$ altitude, increasing to $-5 \%$ per decade at $700 \mathrm{~km}$. A subsequent study was later performed, this time using 5,000 orbiting objects [Emmert et al., 2008]. The results showed an overall density trend of $-2.68 \pm 0.49 \%$ per decade at $400 \mathrm{~km}$ and trends of approximately -5 and $-2 \%$ per decade at solar minimum and maximum respectively.

[6] Marcos et al. [2005] used a Special Perturbations technique to infer long-term thermospheric density changes. Their findings presented a $1.7 \%$ density decrease over the 30-year period 1970-2000. By using satellite drag measurements and a physical general circulation model, Qian et al. [2006] also predicted a 1.7\% secular density change 
over the same 30-year period and then estimate a more severe secular decline if the analyzed time period was extended to the minimum of solar cycle 24 ( 2008).

[7] The aim of this study was therefore to provide a different perspective on thermospheric contraction to make the quantitative analysis more robust. There are many ways of determining atmospheric density, but inferring thermospheric density from satellite drag data is a relatively costeffective way of gathering in situ measurements. Given an initial satellite orbit, our approach is to use an orbital propagator to predict the satellite's state at some time ahead and then to compare that state with TLE data at the same epoch. The difference between the semi-major axis of an initial orbit and that after the orbit propagation is then integrated to obtain an estimate of global average density. This is the approach adopted in our new work, using a bespoke, orbital propagator that includes perturbations due to atmospheric drag, gravitational anomalies, luni-solar gravity effects and solar radiation pressure. This method required accurate estimates of ballistic coefficients; as this information is not contained explicitly in the TLE sets for each satellite, a new way of deriving the ballistic coefficient was developed. In this study, historical satellite data from the past 40 years have been used to infer thermospheric density values over the same period using this new method of ballistic coefficient estimation.

[8] A comparison of the thermospheric densities derived using the method presented in this study, with those derived from an empirical standard atmospheric model, the U.S. NRLMSISE-00 (Naval Research Laboratory's Mass Spectrometry and Incoherent Scatter Radar up to the Exobase, released in the year 2000), is the method by which the longterm trend is established.

\section{Satellite Source Data: TLEs}

[9] The data, from which in situ measurements of atmospheric density are derived, were given by satellite TLE sets. A TLE set consists of various satellite and orbit parameters for a specific satellite at a specific time or epoch. A TLE set is produced by the North American Aerospace Defense Command (NORAD) using a differential correction technique over several observations of a satellite's position to determine an orbit. The program used for this task is the Simplified General Perturbations 4 propagator (SGP4) [Hoots and Roehrhich, 1980]. When a TLE is created, some periodicities are removed to produce an average orbit. To recover the most accurate ephemerides of a satellite's position and velocity using a TLE set, it is necessary to use the SGP4 to convert the TLE set back into an osculating state vector of position and velocity [Hoots and Roehrhich, 1980]; as only the SGP4 can correctly restore the periodicities that were removed during the creation of the TLE set.

[10] The accuracy of satellite ephemerides data provided by the TLEs depends on how they are being used. When determining an orbit directly from the mean orbital elements given explicitly by a TLE set, the accuracy is relatively low and produces an ephemeris error of the order of tens of kilometers. This is due to the elements in the TLE being given as a mixture of Kozai and Brouwer values [Vallado, 1997; T. Payne, U.S. Air Force Space Command, private communication, 2009]. These values are deduced by removing periodicities that naturally occur in a satellite's orbit to give mean, not osculating values. An osculating orbit is the instantaneous Keplerian orbit a satellite would follow around a point mass if all perturbing forces were removed. In the form of osculating state vectors the TLEs modestly provide satellite ephemerides to an accuracy of hundreds of meters to a few kilometers (D. A. Vallado, Center for Space Standards and Innovation, private communication, 2009). The precision of the Kozai mean motion, which is used to determine the semi-major axis, is given in units of revolutions per day to eight decimal places. This implies an accuracy of semi-major axis of approximately $10^{-5} \mathrm{~km}$ at worst case. This means that the errors associated with the determination of the change in semi-major axis, the parameter used in this study, are insensitive to errors in the numerical precision intrinsic to the TLE sets. Therefore, the main source of error is in the determination of the osculating state vector.

[11] Another deficiency of TLE data is that each set has varying accuracy of unknown magnitude. This means there is no covariance carried over with the TLE data (Vallado, private communication, 2009), hence making the error difficult to identify and reduce. With large scale use of TLE data, this introduces a significant source of error into the final results. However, by increasing the temporal separation of the TLE sets used for each satellite, trends in orbit evolution can be identified with sufficient accuracy to enable a quantitative analysis.

[12] Over the four decades analyzed in this study, the TLE data for each satellite were filtered to give 10-day epoch separations. This epoch separation was determined experimentally to obtain an optimum value of signal-to-noise ratio. Increasing the TLE-TLE separation increases the magnitude of the change in semi-major axis due to atmospheric drag. This increase in signal magnitude strengthens the signal under investigation compared with the ephemeris errors of the TLE sets. It was found that using a 10-day epoch separation was sufficient to provide a high enough resolution to highlight periodic density variations, yet long enough to reduce ephemeris noise to a level such that a trend could be derived over the historical period. In general, using a TLE set to predict a satellite's orbit evolution for much beyond 5 days into the future does not provide accurate ephemeris data. However, the semi-major axis is a parameter that is less sensitive to the errors associated with TLE ephemerides. Therefore, even though the TLE sets in themselves cannot provide accurate long-term orbit evolution data, they can be used as initial conditions to analyze other orbital parameters, such as the semi-major axis.

[13] For this study, TLE sets from 41 satellites were used covering the years from 1970 to 2010. Their designations and orbital characteristics are shown in Table 1. As the thermosphere extends from an altitude of $90 \mathrm{~km}$ up to 600 $\mathrm{km}$ (dependent on solar activity), any TLE data from orbiting objects consistently passing through this region during the 40-year historical period was potentially useful. However, many satellites considered for inclusion had either incomplete or low temporal fidelity TLE data during the early part of the 1970s and so could not provide useable long-term data sets. Additionally, some satellite's TLE data needed further filtering to ensure a coherent tracking of the same object. In some, rare, cases an orbiting object was 
Table 1. Physical and Orbital Characteristics of the 41 Satellites Used in This Study

\begin{tabular}{|c|c|c|c|c|c|}
\hline $\begin{array}{l}\text { Satellite ID } \\
\text { Number }\end{array}$ & $\begin{array}{c}\text { Historical } \\
\text { Period Covered }\end{array}$ & $\begin{array}{c}\text { Ballistic } \\
\text { Coefficient }\left(\mathrm{m}^{2} / \mathrm{kg}\right)\end{array}$ & $\begin{array}{l}\text { Associated Density } \\
\text { Altitude Range (km) }\end{array}$ & Eccentricity Range & $\begin{array}{c}\text { Average } \\
\text { Inclination (deg) }\end{array}$ \\
\hline $00060^{\mathrm{a}, \mathrm{b}}$ & $1970-2010$ & 0.0275 & $461.5-368.6$ & $0.1133-0.0194$ & 49.94 \\
\hline $00063^{\mathrm{b}}$ & $1970-2010$ & 0.0184 & $627.1-453.6$ & $0.0087-0.0012$ & 48.55 \\
\hline $00165^{\mathrm{b}}$ & $1970-2010$ & 0.0683 & $746.5-525.5$ & $0.0059-0.0000$ & 47.92 \\
\hline $00229^{\mathrm{b}}$ & $1970-2002$ & 0.0667 & $715.0-232.2$ & $0.0097-0.0006$ & 48.29 \\
\hline $00614^{\mathrm{a}, \mathrm{b}}$ & $1970-2010$ & 0.0146 & $382.7-331.1$ & $0.2097-0.0925$ & 82.09 \\
\hline $00750^{\mathrm{b}}$ & $1970-2010$ & 0.0865 & $455.1-403.1$ & $0.3154-0.1938$ & 60.85 \\
\hline 01335 & $1970-2010$ & 0.1051 & $675.7-606.3$ & $0.0944-0.0531$ & 56.13 \\
\hline $01370^{\mathrm{b}}$ & $1970-2004$ & 0.1499 & $638.8-316.8$ & $0.0808-0.0005$ & 56.07 \\
\hline 01616 & $1970-2010$ & 0.0237 & $459.7-417.6$ & $0.1778-0.1185$ & 144.23 \\
\hline 01685 & $1970-2010$ & 0.0455 & $763.3-510.4$ & $0.0044-0.0000$ & 32.32 \\
\hline $01808^{\mathrm{b}}$ & $1970-2002$ & 0.1611 & $564.6-317.6$ & $0.1435-0.0005$ & 79.80 \\
\hline $01843^{\mathrm{b}}$ & $1970-2002$ & 0.0127 & $634.1-207.6$ & $0.0131-0.0004$ & 64.98 \\
\hline 01857 & $1970-2010$ & 0.0696 & $742.2-555.8$ & $0.0101-0.0016$ & 32.03 \\
\hline 01981 & $1970-2010$ & 0.0376 & $710.5-496.0$ & $0.0038-0.0000$ & 32.44 \\
\hline $02016^{\mathrm{b}}$ & $1970-2010$ & 0.0350 & $554.3-523.2$ & $0.1386-0.1195$ & 34.11 \\
\hline $02129^{\mathrm{b}}$ & $1970-2002$ & 0.0490 & $637.8-181.7$ & $0.0220-0.0009$ & 98.30 \\
\hline $02153^{\mathrm{b}}$ & $1970-2010$ & 0.0359 & $556.6-527.1$ & $0.1511-0.1313$ & 79.78 \\
\hline $02389^{a, b}$ & $1970-2010$ & 0.0189 & $404.3-360.1$ & $0.2274-0.1405$ & 81.45 \\
\hline $02622^{\mathrm{b}}$ & $1970-2002$ & 0.0264 & $646.8-254.1$ & $0.0103-0.0003$ & 93.39 \\
\hline $02643^{\mathrm{a}}$ & $1970-2010$ & 0.0251 & $527.5-496.3$ & $0.2401-0.2248$ & 99.11 \\
\hline $03019^{\mathrm{b}}$ & $1970-2010$ & 0.0506 & $414.8-284.6$ & $0.6937-0.6327$ & 26.34 \\
\hline 03038 & $1970-2002$ & 0.0098 & $558.2-179.8$ & $0.0308-0.0001$ & 64.07 \\
\hline 03462 & $1970-2010$ & 0.2163 & $687.4-597.0$ & $0.0979-0.0572$ & 56.31 \\
\hline $03523^{\mathrm{b}}$ & $1970-2010$ & 0.0256 & $681.2-566.0$ & $0.0058-0.0012$ & 32.05 \\
\hline $03524^{\mathrm{b}}$ & $1970-2001$ & 0.1889 & $639.1-199.4$ & $0.1007-0.0005$ & 62.29 \\
\hline $03553^{\mathrm{b}}$ & $1970-2001$ & 0.1284 & $593.1-246.8$ & $0.1021-0.0013$ & 62.33 \\
\hline 03608 & $1970-2004$ & 0.1633 & $630.0-275.5$ & $0.1027-0.0023$ & 62.26 \\
\hline $03717^{\mathrm{b}}$ & $1970-2010$ & 0.0899 & $575.3-418.7$ & $0.1072-0.0158$ & 62.53 \\
\hline 03835 & $1970-2001$ & 0.1248 & $614.6-166.4$ & $0.0956-0.0032$ & 62.32 \\
\hline 04053 & $1970-2010$ & 0.0167 & $646.3-421.2$ & $0.0048-0.0000$ & 81.19 \\
\hline $04119^{\mathrm{b}}$ & $1970-2010$ & 0.0063 & $310.1-260.7$ & $0.2779-0.1025$ & 30.35 \\
\hline $04221^{\mathrm{b}}$ & $1970-2002$ & 0.0155 & $633.3-188.6$ & $0.0051-0.0000$ & 81.18 \\
\hline $04330^{\mathrm{a}, \mathrm{b}}$ & $1970-2010$ & 0.0225 & $432.9-383.2$ & $0.1701-0.0818$ & 102.83 \\
\hline $04726^{\mathrm{b}}$ & $1970-2003$ & 0.0282 & $382.4-190.1$ & $0.2636-0.0019$ & 31.08 \\
\hline $04849^{\mathrm{b}}$ & $1970-2001$ & 0.1527 & $643.5-314.5$ & $0.0996-0.0014$ & 62.84 \\
\hline $04940^{\mathrm{b}}$ & $1971-2005$ & 0.0141 & $642.7-228.7$ & $0.0048-0.0000$ & 81.21 \\
\hline 05977 & $1970-2001$ & 0.1588 & $654.1-179.6$ & $0.1021-0.0005$ & 62.79 \\
\hline 05998 & $1970-2010$ & 0.0345 & $442.1-238.1$ & $0.7274-0.6114$ & 29.58 \\
\hline $06073^{\mathrm{b}}$ & $1970-1999$ & 0.0347 & $407.8-222.8$ & $0.7222-0.5646$ & 28.16 \\
\hline 14756 & $1972-2010$ & 0.0035 & $257.0-215.4$ & $0.4193-0.2195$ & 52.17 \\
\hline
\end{tabular}

${ }^{\text {a }}$ The satellites that were used in the original study by Keating et al. [2000].

${ }^{\mathrm{b}}$ The satellites that were used in the first study by Emmert et al. [2004].

mistaken for another satellite with a listed ID number and its velocity vector exhibits unnatural behavior as the TLE sets described the different objects.

[14] As atmospheric drag is one of the most significant perturbations affecting satellites in LEO, in general the semi-major axis degrades over time causing the perigee altitude, among other parameters, to secularly decrease. Therefore, in order to derive a model of long-term, altitudedependent thermospheric density change, it was necessary to have multiple satellites orbiting within a similar altitude range. This ensured that reliable density data could be derived over the altitude and time span under investigation. Figure 1 shows the range of orbit altitudes covered by the satellites used in this study.

\section{Methodology}

\subsection{Detecting Density Variations}

[15] To infer changes in local atmospheric density using data provided by the TLEs required a processing method. In their raw form, the TLEs do not explicitly offer any information about atmospheric density, or changes caused thereby. By analyzing the orbit parameters, given by a time series of TLEs, conclusions about physical processes acting upon the orbiting satellite can be made. This is how observational data of thermospheric density was derived and then used to compare with empirical model estimates provided by the NRLMSISE- 00 .

[16] One way to compare observational density data with an empirical atmospheric density model, such as the NRLMSISE-00, is by estimating absolute values of density. Doing this requires a sophisticated method to accurately process the TLE data, such as that described by Picone et al. [2005]. However, the TLEs have inherently low accuracy, as previously described, and so estimating absolute density values can be complicated and involved. If some other measureable parameter could be analyzed through simpler methods that could still be used to infer long-term density changes then the data gathering process would be more efficient. Also, gathering data about the same phenomena in 


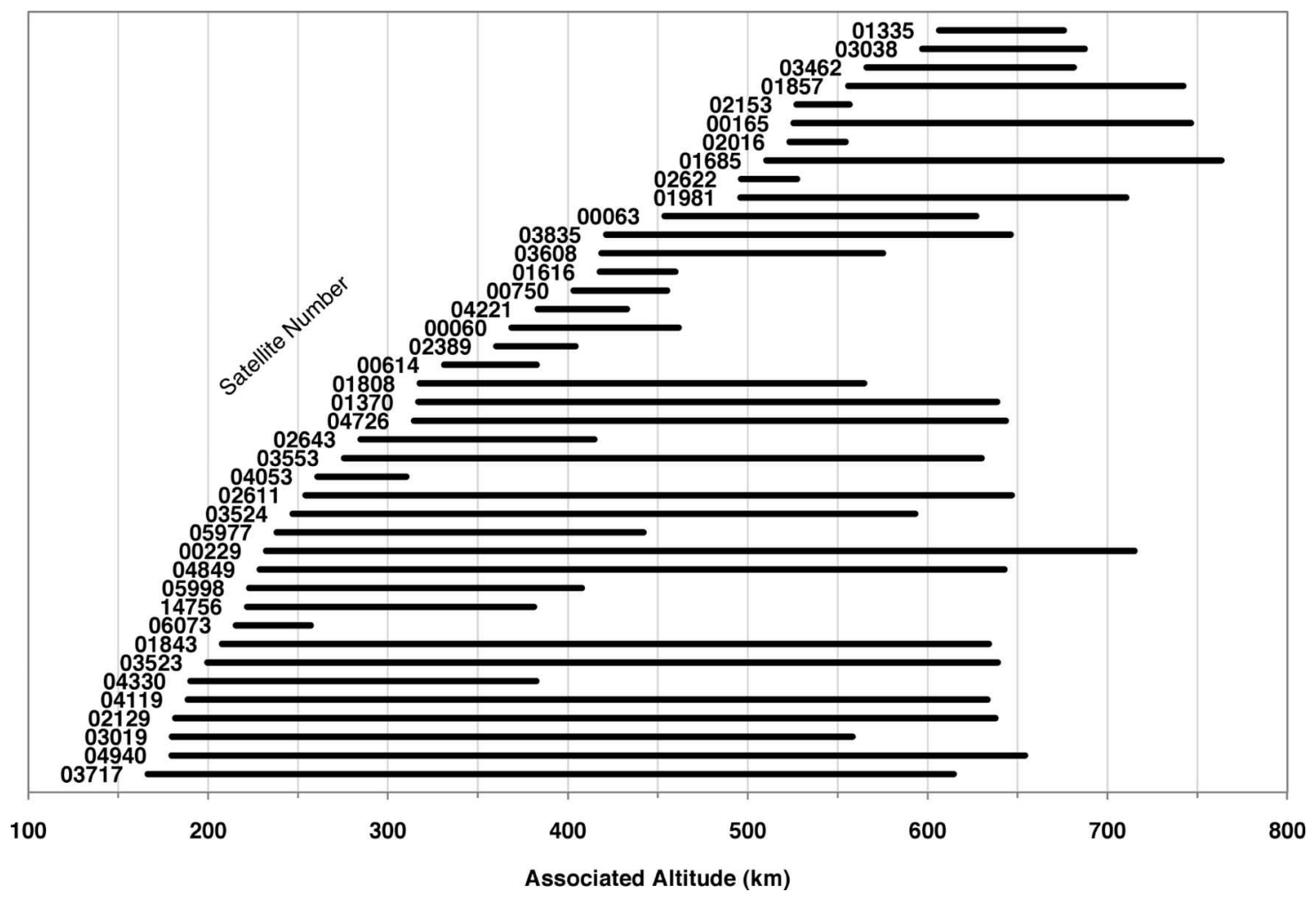

Figure 1. The range of altitudes covered by the 41 satellites used in this study, ordered by associated density altitude: the altitude at which the changes in semi-major axis were attributed to atmospheric drag.

different ways adds to knowledge base of the field. The method used in this study avoids the issues associated with deriving absolute density values by comparing just the effects caused by atmospheric drag, and hence density, on a satellite's orbit. Therefore, in order to deduce a trend, a comparison between observational data and that from numerically derived data needs to be made as given by

$$
\zeta=\frac{\rho}{\rho^{*}},
$$

where $\rho$ denotes the density derived from the observational TLE data and $\rho^{*}$ being the density derived numerically.

[17] Atmospheric drag has a braking effect on a satellite. This process results in a reduction of the semi-major axis of the satellite's orbit. From theory by King-Hele [1987], the local atmospheric density at a point in time on an orbit can be defined by

$$
\rho=\frac{-\mu \dot{a}}{a^{2}|v|^{3} \delta}
$$

where $a$ is the semi-major axis, $v$ is the satellite's velocity relative to the atmosphere, $\delta$ is the satellite's ballistic coefficient and $\mu$ denotes the gravitational constant of the Earth (398600.4415 $\mathrm{km}^{2} \mathrm{~s}^{-3}$ [Montenbruck and Gill, 2005]). For the particular epoch that equation (2) represents, all the parameters are known except the rate of change of $a$ with respect to time, $\dot{a}$. The rate of change of semi-major axis is the parameter by which atmospheric density is analyzed in this study.
[18] As we used a time series of TLEs for the observational data, at epochs of 10 days apart, $\dot{a}$ could only be calculated using

$$
\dot{a}=\frac{a_{2}-a_{1}}{\Delta t}=\frac{\Delta a}{\Delta t},
$$

where the subscripts, 1 and 2, denote the semi-major axis at the epoch of the first TLE and second TLE respectively; and $\Delta t$ is the time between the two epochs (10 days). This gives an average rate of change of $\dot{a}$ and so only an average density value can be derived. In order to calculate equation (1) we need two estimates of density, $\rho$ and $\rho^{*}$. From equations (2) and (3) these density estimates rely on knowledge of the change in the semi-major axis from the TLE data and the numerically derived data from the orbit propagation using the NRLMSISE-00 as its atmospheric density model. Therefore, by combining equations (1), (2), and (3) and simplifying gives

$$
\zeta=\frac{a_{2(T L E)}-a_{1(T L E)}}{a_{2(N U M)}-a_{1(T L E)}}=\frac{\Delta a_{T L E}}{\Delta a_{N U M}}
$$

where the subscripts 'TLE' and 'NUM' denote the total change in semi-major axis from the TLE data and that which is numerically predicted by the orbital propagator using the NRLMSISE-00 atmospheric density model respectively.

[19] To calculate $\Delta a_{T L E}$, the semi-major axes from the consecutive TLE sets needed to be derived. The TLEs do not explicitly give the value of semi-major axis, although 
they do provide a form of the mean motion, $n_{k}$, that can be used to give an estimate of the semi-major axis

$$
a_{T L E}=\sqrt[3]{\frac{\mu}{n_{k}^{2}}} .
$$

The subscript ' $\mathrm{k}$ ' denotes the Kozai mean motion. This is the form used in TLE sets that has had certain orbital periodicities averaged out. However, the secular change presented by the Kozai mean motion is identical to the secular change derived using its osculating state vector equivalent, providing the time between the TLE sets is approximately 10 days or greater. For this reason, using the Kozai mean motion to represent secular effects of atmospheric drag is ideal for the method used in this study; as it requires only the processing shown by equation (5).

[20] Using the TLE data and the Kozai mean motion provides the observational source of thermospheric density. To compare this with model density data we computationally simulated satellite orbit evolution using density estimates as predicted by the empirical atmospheric model, the NRLMSISE-00. As the calculated density values from the NRLMSISE-00 model vary with time, space, solar radio flux and geomagnetic activity, the contribution to the variations in the derived density ratios from equation (1) due to these parameters should be mostly, if not completely, removed. This approach allows a faster analysis of the output data as most natural perturbations affecting the state of the atmosphere will have previously been included.

[21] From the same TLE sets as those used for the observational data, a satellite's osculating state vector of position and velocity were obtained via the SGP4 propagator and used as initial conditions in our orbital propagator. The satellite's state vector was then propagated for precisely the same duration as that between the epochs of the consecutive TLEs.

[22] Again from theory by King-Hele [1987] the rate of change of the semi-major axis from a specific perturbation can be given by

$$
\dot{a}=\frac{2 a\left|v_{E}\right|}{\mu}\left(\ddot{r}_{\text {pert }}\right),
$$

where $\ddot{r}_{\text {pert }}$ is the perturbing acceleration vector, in this case, atmospheric drag and $\boldsymbol{v}_{E}$ is the velocity vector relative to the center of the Earth. The acceleration due to atmospheric drag is given by

$$
\ddot{r}_{\text {drag }}=\frac{1}{2} \rho|v|^{2} \frac{C_{D} A}{m},
$$

where $C_{D}$ is the drag coefficient of the satellite, $A$ is the cross-sectional area presented orthogonal to the velocity vector and $m$ is the satellite's mass.

[23] Combining equations (6) and (7) and integrating yields the total change in the semi-major axis, as numerically predicted by the orbital propagator giving

$$
\Delta a_{N U M}=\int_{T L E 1}^{T L E 2} \dot{a} \mathrm{~d} t,
$$

Having obtained the parameters required to estimate a density ratio, the next important step was to assign it to an associated density altitude. Over a satellite's orbit that enters the atmosphere, the change in the semi-major axis caused by atmospheric drag can be attributed to a particular height relative to the perigee altitude [King-Hele, 1987]. The height depends on a parameter given by

$$
z=\frac{a e}{H}
$$

where $e$ is the eccentricity of the satellite's orbit and $H$ is the density scale height of the atmosphere at the perigee altitude. Assuming $z$ is greater than 3 , the associated density altitude can be assumed to act at approximately half the scale height above the perigee altitude [King-Hele, 1987]. For satellite orbits with $z<3$, the associated density altitude was set equal to the perigee altitude. As some satellite orbits degraded such that their $z$ value was initially greater than 3 and after the historical time span reduced to less than 3 , there arose a discontinuity as to how the associated altitude was assigned. However, in practice when the value of $z$ approaches 3 , the associated altitude asymptotically approached the perigee altitude, thus allowing a smooth transition between phases.

[24] This procedure enables the use of data from satellites with orbital eccentricities of less than one. The range of altitudes for which a satellite will experience drag will increase with orbital eccentricity. The average density encountered by a satellite via atmospheric drag will be averaged to the particular value used when initially creating the TLE sets. By using the approach described above, the average density experienced by a satellite through a range of altitudes can be represented by a density value at a particular location. Furthermore, the procedure used in this study to infer long-term thermospheric changes uses a method of relative change and not the calculation of absolute density values. Therefore, the comparison parameter, $\zeta$, will continue to provide an accurate representation of the relative integral of atmospheric drag as it is calculated from many contributions over the entire orbit path, and hence range of altitudes, traversed by the satellite.

[25] By repeating the process described above using a time series of TLE sets for each satellite over the entire historical time span, the density ratios given by $\zeta$ were used to show long-term trends in atmospheric density variations. As outlined in this section, an orbital propagator was required to derive the numerically estimated density variations as well as an accurate estimate of a satellite's ballistic coefficient. Descriptions of both the orbital propagator, the method of deriving accurate ballistic coefficient estimates and the results using this method are detailed in the following sections.

\subsection{Bin Sizing}

[26] To perform a quantitative analysis of the data, the derived density ratios needed to be grouped within altitude and temporal bins. Owing to the error noise of the resulting data, the temporal bin resolution was set at 1 year. With regards to altitude, we chose a bin size that would represent a constant percentage change in density. As atmospheric density reduces with increasing altitude at an approximately exponential rate, a metric that reflected this behavior was required. Therefore, a constant fraction of the density scale 


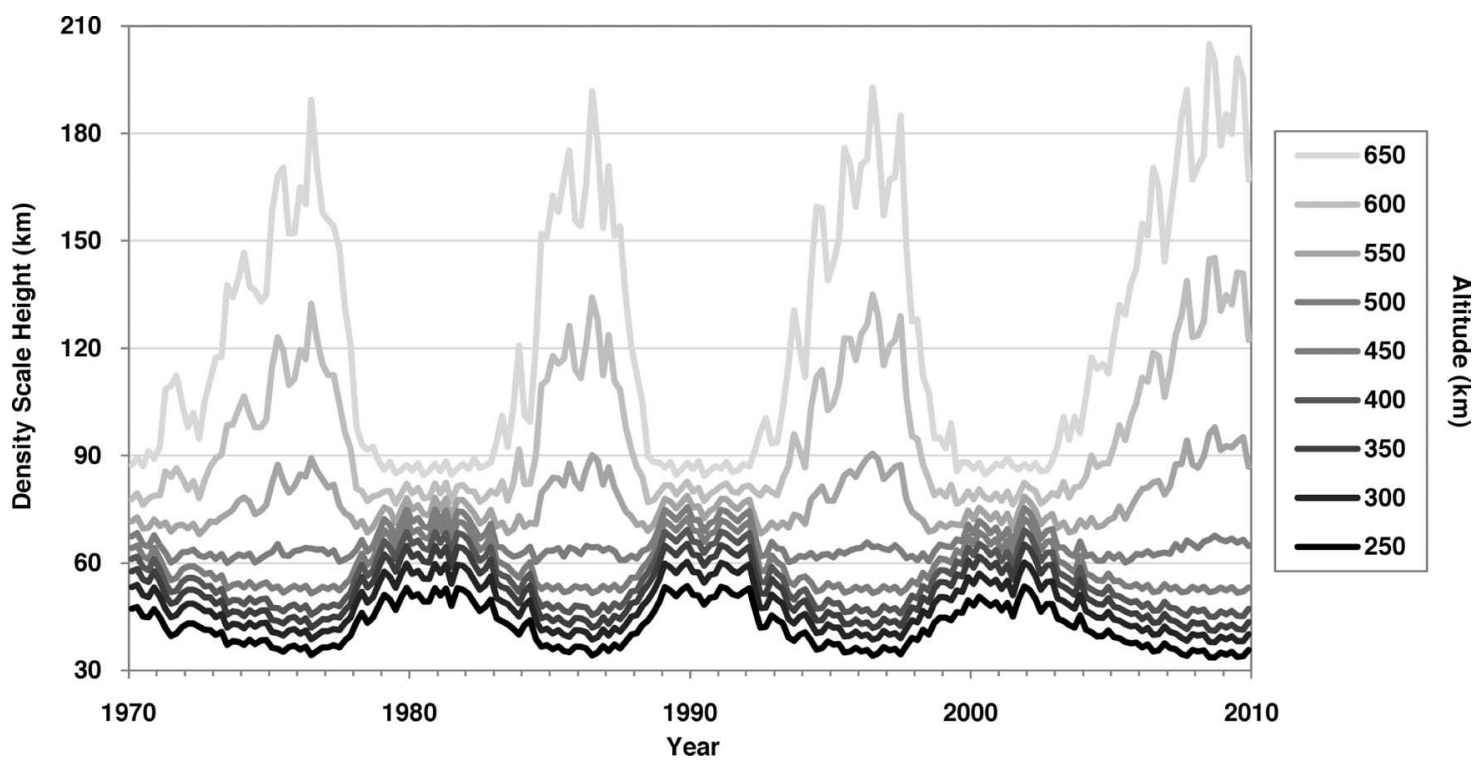

Figure 2. A time history of globally averaged density scale heights from 250 up to $650 \mathrm{~km}$ altitude.

height was chosen: for one density scale height, atmospheric density changes by a factor equal to the mathematical constant $e=2.718$ (base for natural logarithms).

[27] The density scale height depends on a number of physical parameters given by

$$
H=\frac{1}{\frac{M g}{R T}-\frac{2}{r}},
$$

where $M$ is the molecular mass of the local atmospheric composition, $g$ is the gravitational acceleration due to the Earth at the local altitude, $R$ is the universal gas constant $(8.314 \mathrm{~J} / \mathrm{kMol}), T$ is the local atmospheric temperature and $r$ is the distance from the center of the Earth. The NRLMSISE-00 provides estimates of atmospheric molecular mass as well as local atmospheric temperature and so an estimate of the density scale height can be obtained. However, as the state of the atmosphere depends on solar activity, the density scale height at a fixed location is not constant. Figure 2 shows the variation in density scale height across a range of altitudes for the duration of the historical period analyzed in this study.

[28] To determine a suitable fraction of the density scale height to be used as a vertical bin size, a trade-off was necessary between the number of density ratios available and the change in density covered by one density scale height. We decided that an altitude bin size of $0.5 \mathrm{H}$ would provide the optimum balance of sufficient number of density ratios without an excessive change in density: approximately $65 \%$.

[29] The bin coverage encompasses density ratios derived over altitudes extending $0.25 \mathrm{H}$ above and below a particular altitude. Figure 3 demonstrates the altitude coverage resulting from using this method of bin sizing over the historical period. We applied this method of bin sizing at 10 $\mathrm{km}$ increments between the altitudes of 200 and $600 \mathrm{~km}$. This meant that there was some overlap between the bins and so some data points contributed to the derived longterm trends via more than one altitude bin.

\section{Orbital Propagator}

[30] To process the data given in the TLE sets and predict the orbital evolution of the 41 satellites, an Earth orbital propagator for thermospheric analysis (AETHER) was written in the computing language $\mathrm{C}++$. Perturbations from Earth gravitational anomalies are modeled using spherical harmonics up to the order and degree 20, using coefficients from the Joint Gravity Model 3 (JGM3) [Tapley et al., 1996]. Atmospheric drag is predicted using the NRLMSISE-00 empirical atmospheric density model and assumed to corotate with the Earth. Other perturbations include gravitational effects from the Moon and Sun as well as Solar Radiation Pressure (SRP), modeling an oblate conical Earth shadow.

[31] The NRLMSISE-00 empirical atmospheric model is used to provide an estimate of local atmospheric density.

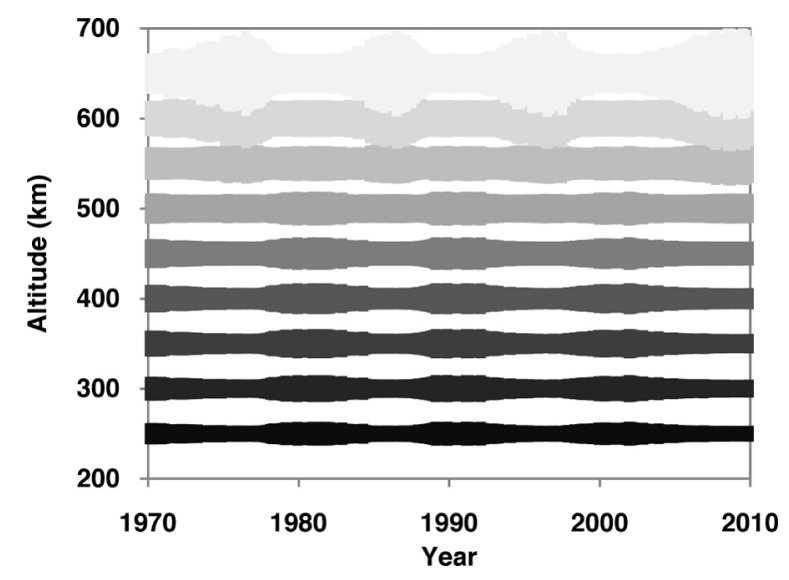

Figure 3. The historical variation of bin sizing due to varying density scale height shown at sample altitudes. 
This model was published in the year 2000 and since then new empirical density models have become available that provide greater accuracy and precision when predicting local atmospheric density. However, the more recent models all require certain solar and geomagnetic indices that only became available in recent years. Therefore, this study required a model that was compatible with the indices available during the historical period analyzed in this study.

[32] It is still possible to infer long-term density changes using a less accurate atmospheric model compared to more up-to-date models. The method employed in this study circumnavigates problems associated with errors in the model densities from the NRLMSISE- 00 by analyzing only relative changes in the atmospheric density as opposed to absolute values.

[33] When calculating atmospheric density the NRLMSISE00 empirical model requires various inputs: satellite geocentric position; date and time of day; solar flux data in the form of the $\mathrm{F} 10.7 \mathrm{~cm}$ radio flux and the geomagnetic index, $\mathrm{a}_{\mathrm{p}}$, for varying times prior to the date given. The daily indices for both the F10.7 cm solar flux and geomagnetic index were given by National Oceanic and Atmospheric Administration (NOAA) [Erwin, 2007]. AETHER linearly interpolates between the given indices to provide a continuous value between the available daily data. This was done to eliminate input step changes when the orbital integration time moves over one day and the F10.7 flux index changes from one value to the next. Similarly, the geomagnetic $a_{p}$ index values were linearly interpolated to provide a moving average between the published 3-hourly indices.

[34] To calculate the gravitational effects of the Moon and Sun, as well as obtaining the directional Sun vector for calculating SRP, their positions are obtained from NASA's Horizons [Yeomans, 2009]. This system provides accurate ephemerides for many solar system bodies including the Moon and Sun for any date between 3,000BC and 3,000AD. For each propagation step, AETHER calls an internal database of Moon and Sun ephemerides gathered from Horizons and then calculates their gravitational effects using an expression from Montenbruck and Gill [2005] given by

$$
\ddot{\mathbf{r}}_{\text {moon-sun }}=\frac{\mu_{s}}{s^{3}}\left[-\hat{\mathbf{e}}_{r}+3 \hat{\mathbf{e}}_{s}\left(\hat{\mathbf{e}}_{r} \hat{\mathbf{e}}_{s}\right)\right],
$$

where the subscripts ' $r$ ' and ' $s$ ' denote the parameters specific to the satellite and perturbing body respectively.

[35] To test the implementation of equation (11), propagations by AETHER were compared to the same propagations using the Astrogator propagator from the Analytical Graphics Inc. Satellite Tool Kit software [Analytical Graphics, Inc., 2009]. Sample propagations were performed to obtain various orientations of the Moon and the Sun relative to the Earth. The satellite orbit used for comparison was an equatorial geostationary configuration. The satellite's altitude was the parameter used to compare the perturbations with the results from AETHER and STK. The geostationary orbit configuration was chosen because it is sensitive to gravitational effects from the Moon and Sun. The maximum deviation between the results of AETHER and STK was approximately $1 \mathrm{~km}$. The semi-major axis for a geostationary orbit is approximately $42,000 \mathrm{~km}$. Therefore the results indicate a satisfactory relative error of approximately $0.002 \%$ in satellite altitude.
[36] The effect SRP has on a satellite orbit's semi-major axis can be approximately five orders of magnitude less than that of atmospheric drag at altitudes of $200 \mathrm{~km}$ but approximately equal in magnitude during times of low solar activity and at altitudes of $600 \mathrm{~km}$. Therefore, errors associated with incorrect modeling of SRP could be significant for satellite orbits considered in this study. However, the inclusion of SRP in AETHER's acceleration model is important due to indirect effects SRP can have on other perturbations. From Aksnes [1974] the acceleration due to SRP can be approximated by en by

$$
\ddot{r}_{s r p}=-P C_{r} \frac{A}{m} \frac{\mathrm{AU}^{2}}{r_{s u n}^{2}},
$$

where the pressure of solar radiation, $P$, is assumed to be $4.56 \times 10^{-6} \mathrm{Nm}^{-2}$ [McCarthy, 1996], $r_{\text {sun }}$ is the position of the Sun relative to the satellite and AU is a standard astronomical unit. The reflectivity coefficient, $C_{r}$, can take any value between 0 and 2. However, results by van der Ha and Modi [1977] show that a value of 1.25 is a suitable approximation for the majority of satellites. The implementation of this SRP model was tested using the example cases provided in the paper by Aksnes [1974]. The results from AETHER deviated from the published results by a maximum of $50 \mathrm{~m}$ within a $10,000 \mathrm{~km}$ semi-major axis, yielding satisfactory precision.

[37] When performing orbit propagation, AETHER initially requires an osculating state vector provided by the TLEs via the SGP4 propagator. The six-variable osculating state vector is then numerically integrated using a 7th order Runge-Kutta-Fehlberg-Horn (RKFH) method with a $20-\mathrm{s}$ step size. The RKFH method incorporates a continuous step size function that allows an arbitrary final integration step size. This was an important requirement as it was necessary to propagate a satellite's state vector for an equal duration to the epoch separation of the consecutive TLE sets. After each integration step, the osculating state vector and the acceleration due to atmospheric drag were returned by the propagator to enable calculation of the parameters required by equation (6). Using the osculating state vector to provide an estimate of the semi-major axis was done using

$$
a=\frac{\mu|r|}{2 \mu-|r||v|^{2}} .
$$

To validate AETHER, re-entry predictions of satellites nearing their demise were used to gauge the precision of the acceleration models and the ability to accurately predict orbit evolution. The first validation test used three satellites that had previously decayed [Saunders et al., 2009]. The TLE sets from up to 15 days before the satellite's known reentry dates were used as the initial conditions. The results showed excellent consistency in the predicted evolution of the satellite's orbits. However, for these tests, the ballistic coefficients for each satellite were obtained using the $\mathrm{B}^{*}$ number, a parameter given explicitly by the TLEs that is defined to represent a satellite's true ballistic coefficient. In reality, the $\mathrm{B}^{*}$ number is a fitting parameter that is used when deriving the orbit given by the classical orbital elements in the TLEs. These tests highlighted the requirement for a better way to estimate a satellite's ballistic coefficient. 
A new method of estimating accurate satellite ballistic coefficients was developed and the re-entry tests were re-run using the updated ballistic coefficient values. The results predicted a re-entry date to within $1 \%$ of the lead time of 15-days. The next section describes the new method by which an accurate estimate of a satellite's ballistic coefficient can be determined using only the data given by TLE sets.

\section{Ballistic Coefficient}

\subsection{The Estimation Method}

[38] The results of AETHER's validation tests showed it was necessary to have a more accurate method of estimating ballistic coefficients. As the driving force of this work has been to validate a long-term density change using TLE sets, a desired characteristic was that the new method of estimating ballistic coefficients uses only the TLE data. This would eliminate the requirement for multiple independent data sets, as well as simplify the data processing.

[39] There are many ways of estimating a ballistic coefficient. In this study, the ballistic coefficient is defined by the last three terms in equation (7) as

$$
\delta=\frac{C_{D} A}{m} .
$$

For satellites with regular shapes, such as spheres, it is relatively easy to estimate a ballistic coefficient theoretically. With a known mass, projected surface area and satellite surface properties, the drag coefficient can be derived in laboratory conditions. However, real satellites are rarely regular shapes and often vary their projected surface area: either through tumbling or attitude control. Therefore, predicting a real satellite's ballistic coefficient via theoretical means becomes extremely difficult.

[40] There are two main ways of estimating a ballistic coefficient: analytically, using a satellite's documented mass, geometry, surface properties and estimating its projected area, or empirically using data of a satellite's orbit. We employed the latter in our study.

[41] Previous studies have estimated a satellite's ballistic coefficient by using orbit data and applying a batch least squares fit to a series of observations. In our method we once again use the TLEs to provide a series of orbital observations and by a similar method to that outlined above, we use changes in the semi-major axis to derive an accurate estimate of ballistic coefficient.

[42] Via equation (5), the difference in the Kozai mean motion from two consecutive TLEs gives the change in semi-major axis, $\Delta a_{T L E}$. This provides the observational data that is used to derive a value of ballistic coefficient. Using the same TLE sets, AETHER propagates the satellite's orbit from the ephemeris of the first TLE set to the epoch of the next TLE set then numerically derives its own change in semi-major axis, $\Delta a_{A E T}$, which is calculated using equation (8). However, in order for AETHER to propagate the orbit, taking into account atmospheric drag and SRP, a value of the area-to-mass ratio is required. As an initial estimate for $\delta$, the $\mathrm{B}^{*}$ value from the first TLE set is used. In some cases, older TLEs have a $\mathrm{B}^{*}$ value of $+00000-0$ (zero), which is obviously not a valid value and so an arbitrary initial guess of $0.01 \mathrm{~m}^{2} / \mathrm{kg}$ is used. As the area-to- mass ratio forms part of the ballistic coefficient, by assuming an approximate value for the drag coefficient of 2.2 , the area-to-mass ratio is derived from the $\mathrm{B}^{*}$ ballistic coefficient estimate to be used when calculating the acceleration due to atmospheric drag and SRP.

[43] Once the values for $\Delta a_{T L E}$ and $\Delta a_{A E T}$ are obtained, the difference is calculated and stored in a comparison parameter given by

$$
\Delta\left(\delta_{n}\right)=\Delta a_{A E T}-\Delta a_{T L E},
$$

where $\Delta\left(\delta_{n}\right)$ denotes the difference between the change in semi-major axes from the numerical and observational data for a particular value of ballistic coefficient, $\delta_{n}$.

[44] If the comparison parameter yields a significant difference between the observational and numerical values, the propagation is re-run with a revised value of $\delta$ obtained using the Secant method defined by

$$
\delta_{n}=\delta_{n-1}-\frac{\Delta\left(\delta_{n-1}\right)\left(\delta_{n-1}-\delta_{n-2}\right)}{\Delta\left(\delta_{n-1}\right)-\Delta\left(\delta_{n-2}\right)} .
$$

This is repeated until the difference in the observational and numerical results correspond to within a predefined limit, hence making it an iterative process. Once the iterative method to evaluate $\delta_{n}$ has converged, the process is repeated over as many consecutive TLE sets as is required.

[45] A modified form of the Gaussian function, which is then applied to each predicted $\delta$ value, is given by

$$
g(x, \delta)=\frac{1}{n} e^{-\left(\frac{x-\delta}{\delta}\right)},
$$

where $n$ is the number of $\delta$ value estimates in the set $\delta^{*}, x$ is the variable along which the distribution is plotted, and here $\bar{\delta}$ denotes the mean of all the $\delta$ value predictions. Applying equation (17) to the entire set of predicted $\delta$ values (e.g., $\left.\delta_{(1,2, \cdots n)} \in \delta^{*}\right)$ and summing yields the function

$$
G\left(x, \delta^{*}\right)=\frac{1}{n} e^{-\left(\frac{x-\delta_{1}}{\bar{\delta}}\right)}+\frac{1}{n} e^{-\left(\frac{x-\delta_{2}}{\bar{\delta}}\right)}+\ldots+\frac{1}{n} e^{-\left(\frac{x-\delta_{n}}{\bar{\delta}}\right)}
$$

The maxima of the function given in equation (18) gives the most common $\delta$ value of the entire range of predicted ballistic coefficients; and so differentiating equation (18) with respect to $x$ and setting to zero provides the final required value for the satellite's ballistic coefficient. More detail on the accuracy of this method is detailed in the study by A. Saunders et al. (manuscript in preparation, 2011).

\subsection{Long-Term Ballistic Coefficient Variability}

[46] For this study, TLE sets from the years of 1999 and 2000 with a TLE epoch separation of 10 days were used to derive values of ballistic coefficients. Therefore part of the selection criteria for what satellite data were eligible for use in this study was that they must have sufficient 10-day epoch fidelity in their TLE sets during this two-year period.

[47] This particular historical period provided frequently published TLE sets for the majority of satellites. In addition, it was a period when solar activity was neither at maximum nor minimum, therefore representing an 'average' state. 


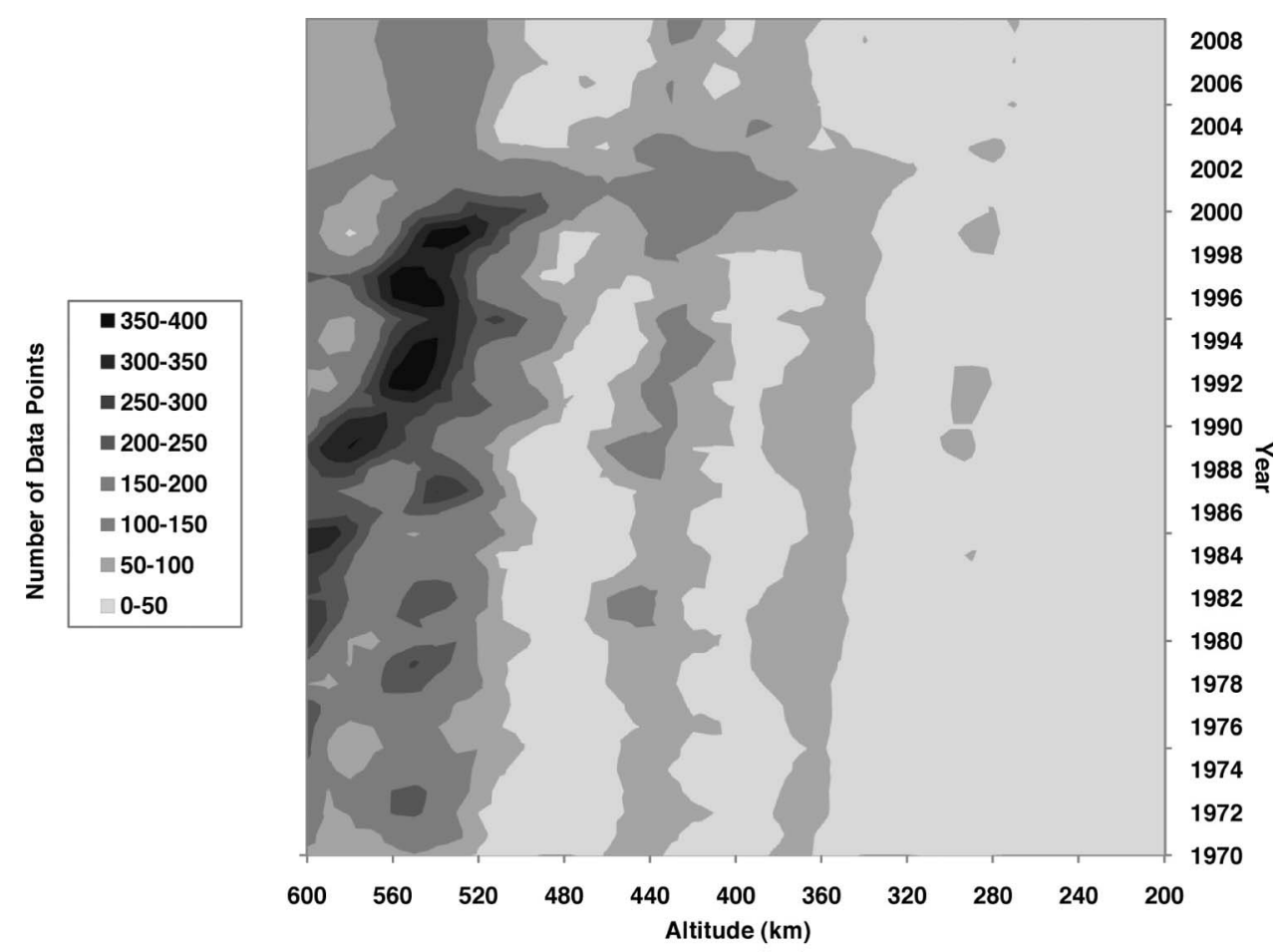

Figure 4. The number of derived density ratios from the TLE sets of the 41 satellites over the 40 years of historical data.

[48] It was important that the estimates of ballistic coefficient were all derived using the same time period. Owing to the information being used for long-term density trend analysis, there needed to be a particular reference period to which the trends would be relative. By making the ballistic coefficient temporally independent we separate the effects of atmospheric drag, and hence the calculation of mean density, over the entire historical period from the estimation of the ballistic coefficient. Therefore, the minimum requirement for eligible satellite data was that its database of TLE sets must cover the historical time span between the years 1999-2000.

[49] One of the parameters that contribute to a satellite's drag coefficient is the atmospheric composition. Depending on what gas species are dominant, the drag coefficient can vary accordingly. As one moves vertically through the atmosphere, the ratios of the different gas species vary approximately according to their molecular mass. This being the case, the drag coefficient can be considered altitude-dependent.

[50] As well as the gas ratios affecting the drag coefficient, some species directly affect the material surfaces of a satellite. Atomic oxygen is one such species; and over time, can adhere to a satellite and alter its surface properties.

[51] Studies have shown that for an object with constant mass, geometry and projected surface area, the drag coefficient within the lower thermosphere $(150-500 \mathrm{~km})$ can reduce by a fraction 0.08 up to 0.15 per $100 \mathrm{~km}$ in altitude for a F10.7 cm solar activity of 200 to $65 \times 10^{-22} \mathrm{~W} / \mathrm{sm}^{2}$ respectively [Moe and Bowman, 2005]. Due to the lack of available results in this area of study, a linear fit using these two datum points was used to provide an approximate altitude dependent multiplier for the estimation of the drag, and therefore ballistic, coefficient.

[52] As the semi-major axis of a satellite orbit in an atmosphere reduces over time, so does its associated density altitude. Therefore, when predicting the effects of atmospheric drag in an orbital propagator, such as AETHER, the variation of atmospheric regime and composition would alter the drag coefficient; and so the variation of ballistic coefficient must be taken into account. For this study, longterm orbit evolution is a key ingredient with orbit decay playing a vital role that needs to be addressed.

[53] By using the average associated density altitude of the satellite orbits during the period of ballistic coefficient determination (1999-2000), a reference point was available from which the values of ballistic coefficients could be varied according to their associated density altitude throughout the historical period used to infer long-term density changes.

[54] From equations (6) and (7), the change in semi-major axis is directly proportional to the drag coefficient and thus the ballistic coefficient. Therefore, adjusting the density ratios obtained from the observational and numerical data, required an altitude and solar activity dependent multiplier given by

$$
\Delta \zeta=0.01\left(\zeta_{a l t}-\delta_{a l t}\right)(-0.000526 F 10.7+0.182185),
$$

where $\zeta_{\text {alt }}(\mathrm{km})$ denotes the associated density altitude, $\delta_{\text {alt }}$ $(\mathrm{km})$ denotes the average associated density altitude during the ballistic coefficient determination period and F10.7 is the solar radio flux $\left(10^{-22} \mathrm{~W} / \mathrm{sm}^{2}\right)$. The numerical values stated in equation (19) are derived from the linear fit of the 


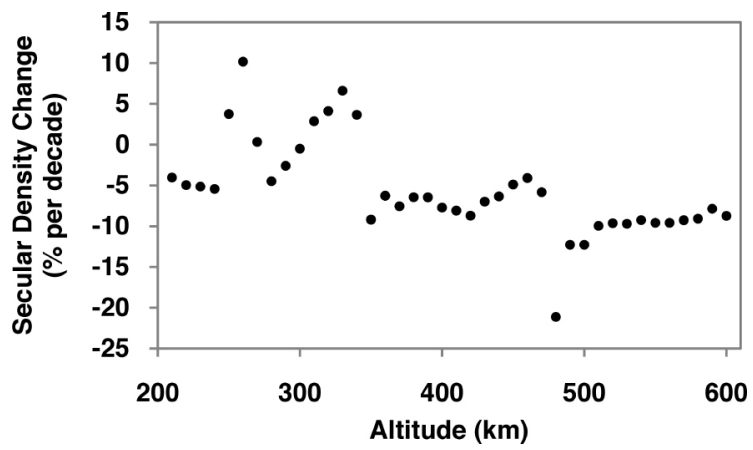

Figure 5. The altitude-dependent secular trend results from a linear regression analysis of the density data.

data supplied by the studies involving the dependence of drag coefficient on altitude [Moe and Bowman, 2005].

\section{Results}

[55] From the TLE data of the 41 satellites over the 40year historical period 44,843 density ratios were obtained. The derived density data were unevenly distributed between the altitudes of 200 and $600 \mathrm{~km}$ as shown in Figure 4. All data points within each bin were averaged to provide one value of density ratio. This reduced the noise in the long term trend signal as well as eliminating any bias in the linear trend analysis caused by an increase in data points from one region of the historical period.

[56] The derived density ratios were grouped into altitude bins positioned at $10 \mathrm{~km}$ altitude intervals, as well as 1-year temporal intervals. This resulted in a time series of 40 averaged density ratios for each altitude bin. Performing a linear regression analysis on each time series of density ratios provided estimates of long-term secular trends. The resulting altitude-dependent trends are shown in Figure 5 for the average change in density per decade.

[57] Some altitude bands showed inconsistent results with the general altitude dependent trend. From investigating the spread of density ratios as shown in Figure 4, regions that returned spurious regression values were identified and removed from further analysis. It was found that density ratios had a reliable signal-to-noise ratio, due to the noise errors of the TLE ephemerides, when there were more than approximately 20 ratios per bin. Anything less than 20 showed inconsistent and spurious results. With the exception of altitudes between 200 and $240 \mathrm{~km}$ spurious results were obtained with regions with less than 20 density ratios. The density ratios derived between the altitudes of 200 and $240 \mathrm{~km}$ were mostly derived from one satellite (ID 06073), which was in a relatively stable orbit. As such, after averaging the density ratios into one-year temporal bins a reliable time series of density ratios were obtained for those altitudes.

[58] During the minimum of solar cycle 23-24, the Sun's activity remained very low for a period of time longer than average. As a result, record-low densities were observed in the thermosphere [Emmert et al., 2010]. When the NRLMSISE-00 model was formulated, it was based on empirical data from observations before the year 2000. More specifically, the data set used to obtain the analytical coefficients for the atmospheric model did not contain density values as low as those observed during the minimum of solar cycle 23-24. Therefore, after correlating solar flux indices with pre 2000 observed density values, the NRLMSISE-00 was based on observations that did not extend to the values witnessed during the solar minimum 23-24. Therefore, the empirical atmospheric model would not accurately predict atmospheric densities during a time when observed densities were reduced below previous records. From the density ratios derived from sample alti-

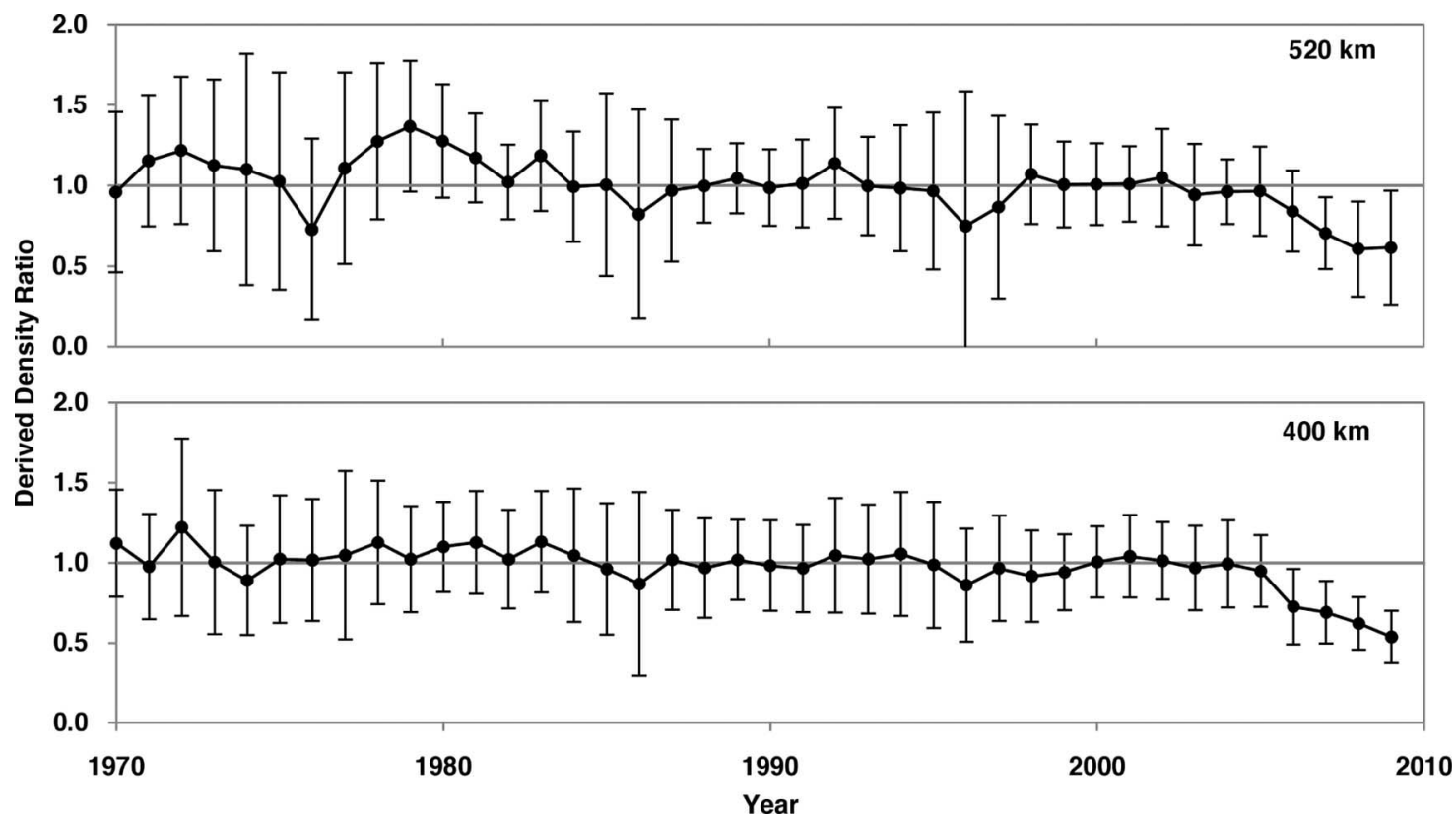

Figure 6. From the derived density ratios within the altitude bins of 400 and $520 \mathrm{~km}$, the anomalies associated with solar cycle minimum 23-24 can be clearly seen. 


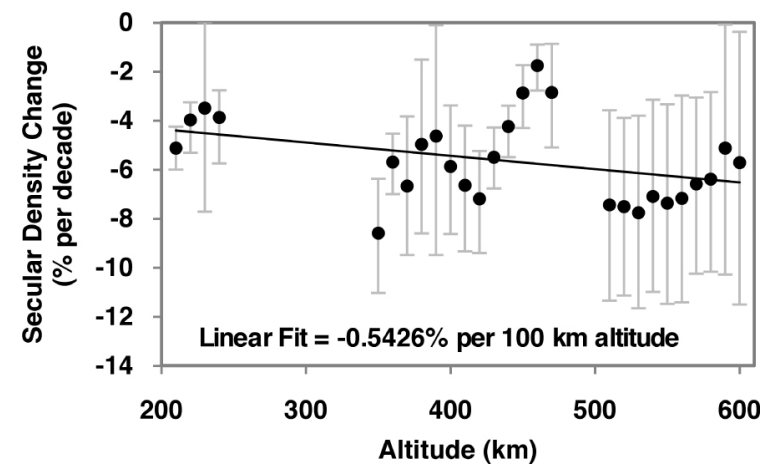

Figure 7. The linear altitude-dependent model of longterm secular density change after removing spurious regression results as well as truncating the historical period at the minimum of solar cycle 23-24. The error bars denote the maximum and minimum trends that could result from the errors presented for each altitude bin as shown in Figure 6.

tudes of 400 and $520 \mathrm{~km}$, a reduction in observed density was apparent during the time of solar cycle minimum 23-24 (2008-2009), as shown in Figure 6. These sample altitudes were shown to demonstrate the difference in errors associated with the density ratio drop during the solar minimum of cycle 23-24. The errors associated with the density trends during this minimum, shown in Figure 6, signify a more certain density drop at $400 \mathrm{~km}$ than at $520 \mathrm{~km}$. However, from the data obtained and shown in Figure 6 it is not possible to definitely say whether the amplitude of the drop is more significant for the lower altitude band. For example, at the altitude of $580 \mathrm{~km}$ the density ratio for 2008 was 0.6544 with an error of $14.4 \%$ above and below this value. The errors above and below the density ratio for 2008 for both the altitude bands of 400 and $520 \mathrm{~km}$ were 16.5 and $35.5 \%$ respectively. Therefore, due to the errors in the results obtained, most significantly due to the errors in the
TLE ephemeris data, no definite conclusion can be drawn from the altitude-dependent behavior of the density drop of the minimum of solar cycle 23-24.

[59] Due to these record-low density observations, the density ratios derived in this study from the period of solar minimum cannot be assumed reliable. Therefore, taking into consideration the spurious regressions results as described in the previous paragraph, in addition to the derived density ratios from the solar minimum of cycle 23-24, the altitudedependent model of long-term secular density change was truncated as shown in Figure 7. A linear best fit was applied to the highly scattered density trend values resulting in an altitude-dependent relationship of $-0.5426 \%$ per $100 \mathrm{~km}$ in altitude. However, the regression coefficient for this line is only 0.1334 therefore only tentatively supporting the altitude dependent relationship. The errors associated with the trend values are shown. These errors are the maximum and minimum trend values that could occur from the possible range of values of the density ratios as denoted by the error bars in Figure 6.

[60] The theory behind thermospheric cooling [Roble and Ramesh, 2002] suggests the concentrations of the local atmospheric gas species are of prime importance. During the 11-year cycle, whereby the atmosphere swells and contracts, the relative composition and density of the atmosphere could increase the reaction rate of energy transfer between the molecules of different gas species. Therefore, it is reasonable to assume that during times of varying solar activity, thermospheric cooling would also vary. The method of linear regression was again applied to the derived density ratios, but this time dividing the data into high and low solar activity: F10.7 $<90$ and $>90$ SU (Standard solar flux units $10^{-22} \mathrm{~W} / \mathrm{sm}^{2}$ ) respectively. The results gave a reduction in thermospheric cooling during times of high solar activity and an increase in cooling during low solar activity as shown in Figure 8. Emmert et al. [2004] also performed an analysis of secular trend variation with solar flux. Their results were spread over several solar flux bins and also

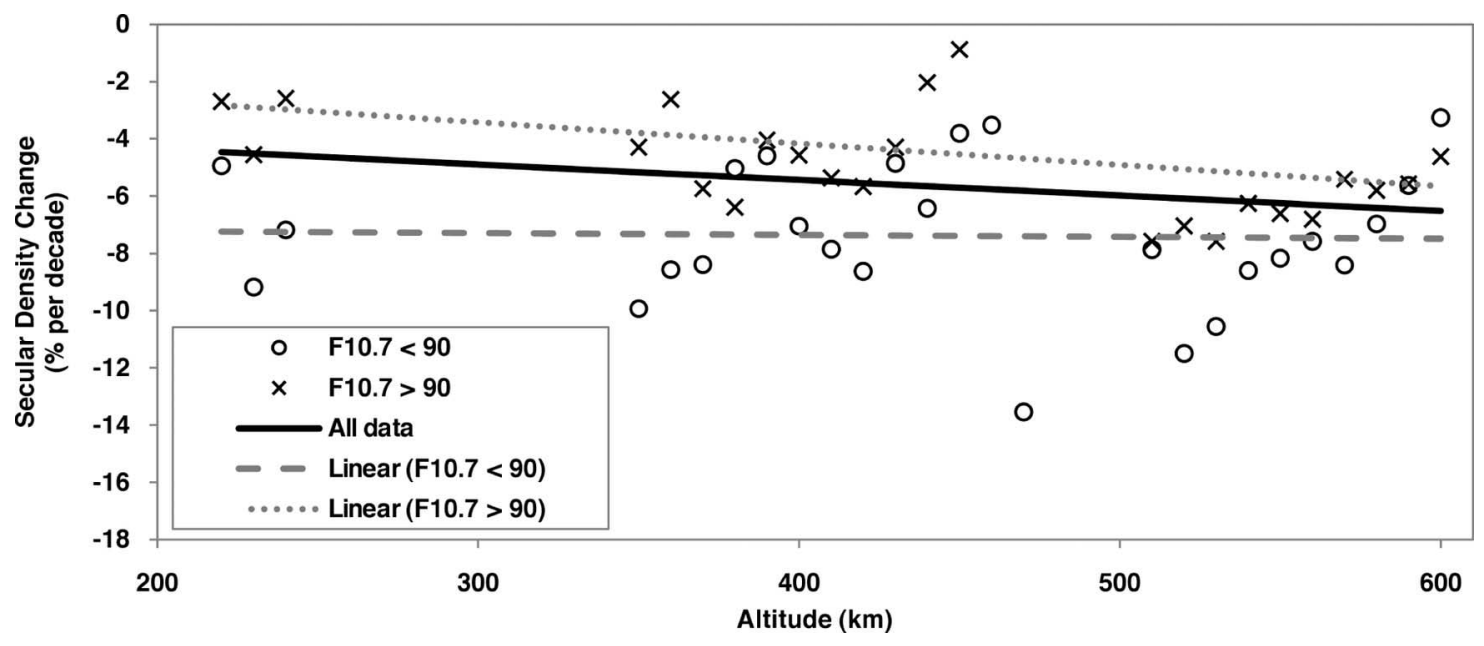

Figure 8. Thermospheric cooling rates during times of high (F10.7 $>90 \mathrm{SU})$ and low $(\mathrm{F} 10.7<90 \mathrm{SU})$ solar activity, plotted against the cooling rates using the combined data set of derived density ratios. Error bars have been omitted from this plot for clarity but are similar in magnitude and behavior to those shown in Figure 7. 


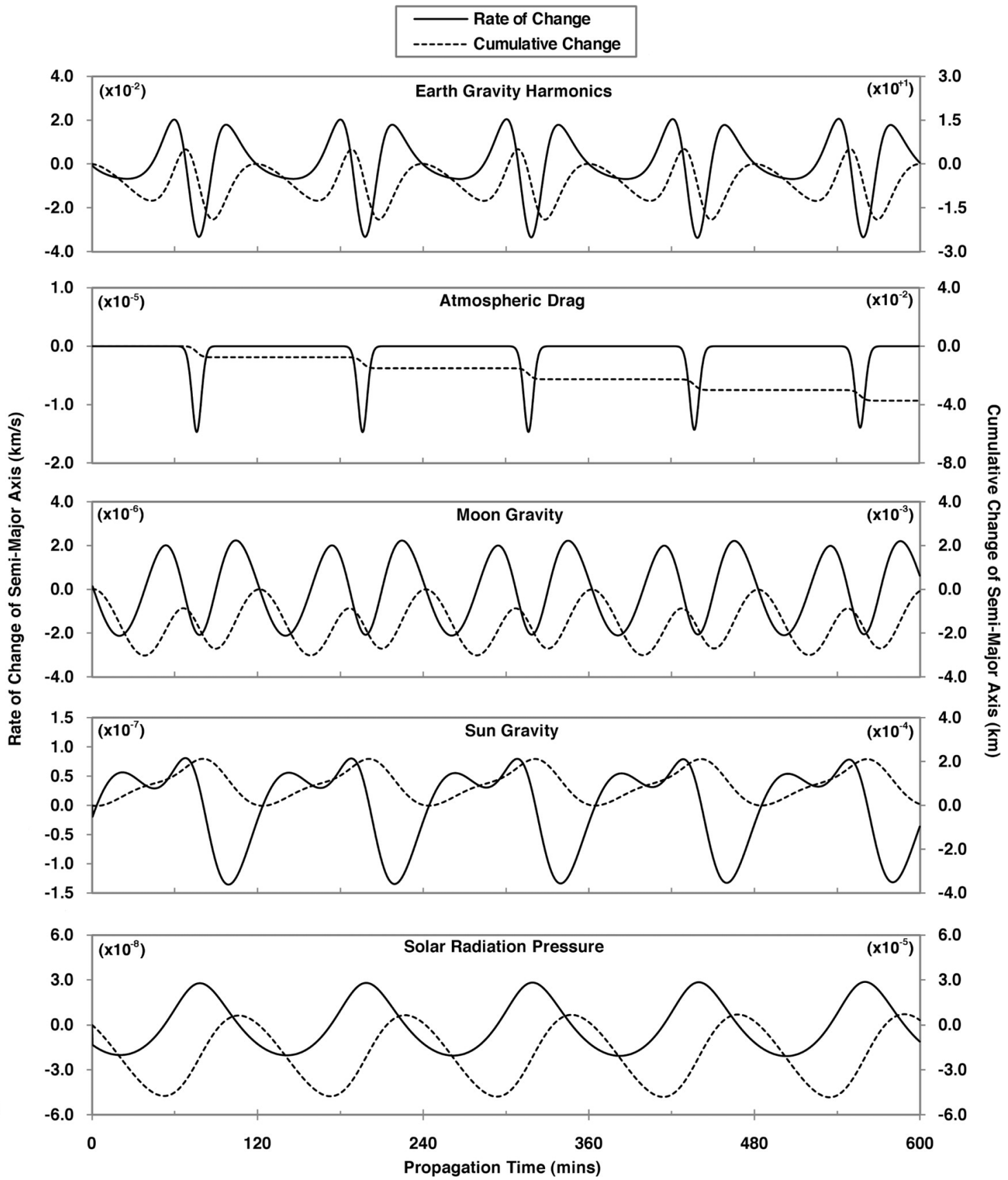

Figure 9. The rate of change and cumulative change in semi-major axis due to the modeled perturbations in AETHER.

showed an increase in the cooling trends during times of low solar activity.

\section{Discussion and Spectral Analysis}

\subsection{Orbital Propagation Errors}

[61] By using a numerical propagator, many assumptions are made about the satellite and perturbations. For this study, the errors associated with most of these assumptions can be easily negated due to their relatively insignificant magnitude. However, other assumptions need to be addressed.

[62] The acceleration model used to estimate satellite orbit perturbations is based on analytical theory. To model the non-spherical geometry of the Earth in terms of its gravitational field, a mathematical formulation using Legendre polynomials was used to simulate the variations in zonal and tesseral gravitational harmonics. To perfectly model the Earth's gravitational field, the order and degree of harmonics included in this model must be infinite. However, obviously it is impossible to achieve such a detailed representation. Therefore, to establish a sensible limit to the detail of the acceleration model, the quantity that is being investigated is the deciding factor. In this study, the change in semi-major axis is the parameter through which all analyses are made. So by investigating the effects of the modeled perturbations on the semi-major axis we were able to 

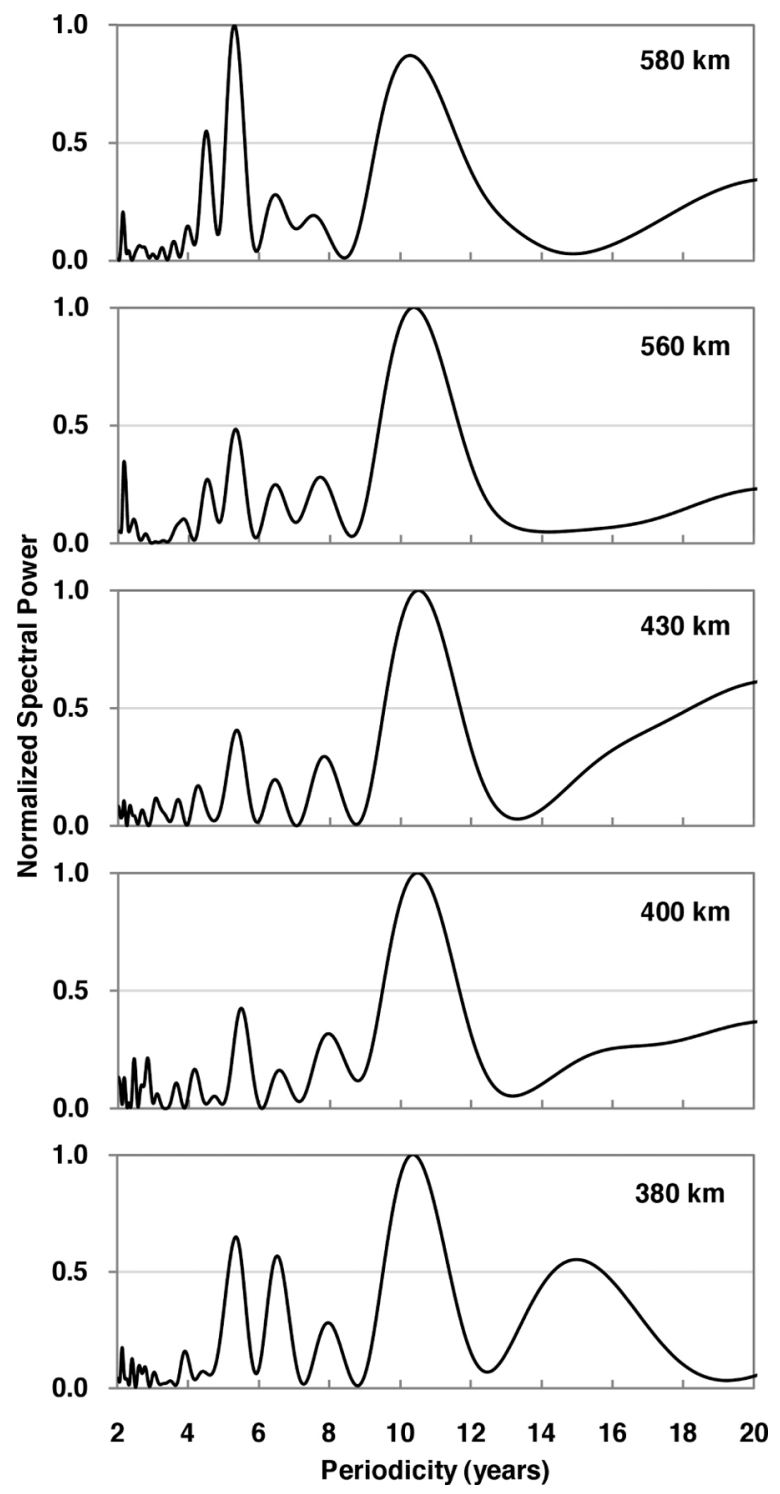

Figure 10. The resulting periodicities from a Fourier spectral analysis of the density ratios of five altitude bins.

determine the limit of detail required for the gravitational potential expansion.

[63] Figure 9 shows the variation and cumulative variation in semi-major axis caused by all the perturbations modeled in AETHER: Earth's gravity, atmospheric drag, the Moon's gravity, the Sun's gravity and SRP. When using the mean motion given in the TLE data, we assumed it was the result of atmospheric drag alone. However, in reality, all perturbations cause a secular change in the semi-major axis; Figure 9 attempts to capture the significance of atmospheric drag in terms of its secular effect. The other plots shown in Figure 9 demonstrate the relative lack of secular effect caused by the other main orbit perturbations. Therefore we can conclude that using the change in semi-major axis as inferred from the mean motion given in the TLE sets is an acceptably precise method.

[64] When approximating atmospheric drag in AETHER, an estimate of local atmospheric density is obtained using the NRLMSISE-00. The state of the atmosphere is largely affected by the irradiance of the sun. More specifically the extreme ultraviolet (EUV) emissions from the sun directly affect the upper atmosphere. Therefore, to model atmospheric density, the magnitude of the EUV emissions would be assumed a necessity. Unfortunately, nearly all the EUV radiation is absorbed in the upper atmosphere and so ground-based observatories are unable to measure the EUV radiation interacting with the upper atmosphere. However, the solar F10.7 cm radio flux does reach the ground and can be used as an approximate proxy to EUV radiation. However, the correlation of F10.7 with EUV is not perfect and so during times of extreme values in the EUV radiation, using the F10.7 indices can cause erroneous results. Liu et al. [2006] performed a study comparing the relationship between F10.7 and EUV radiation using daily averaged data gathered from the Solar EUV Monitor on board the Solar Heliospheric Observatory, launched in 1995. Between the years 1996 and 2005 the relationship between the F10.7 and EUV flux was found to be nonlinear. From a study of a solar EUV flux model, Tobiska and Barth [1990] stated that the correlation coefficient between EUV fluxes and the groundbased F10.7 flux measurement was approximately 0.9.

[65] As this study required the ability to numerically predict atmospheric density over the historical time period, it was necessary to use a model that was compatible with the available solar flux indices (F10.7); only recently with the launch of solar observational satellites has the EUV radiation become directly measureable. Therefore, based on a statistical analysis by Picone et al. [2002], we used the most accurate and complex empirical atmospheric model available, the NRLMSISE-00. With regards to the results obtained in this study, using the NRLMSISE-00 to provide a comparative numerical atmospheric density is acceptable for the majority of the historical time except during times of extreme values of EUV emissions. The only period where this is the case is during the solar minimum of cycle 23-24, where the F10.7 value reduced beyond the previous three solar cycle minima by approximately $5.2 \%$. This is derived from the relation between the minimum of solar cycle 23-43 of $59.3 \mathrm{SU}$ and the average of the previous three solar cycles 20-21, 21-22 and 22-23 that reached 62.5, 61.9 and 62.9 SU respectively. For this reason the density ratios defined during these periods could not reliably contribute to the over density trend was therefore eliminated from our concluding results.

\subsection{Comparison With Previous Studies}

[66] From previous studies into long-term secular changes in atmospheric density, the one that most closely represents the historical and altitude boundaries as investigated in this study was that done by Emmert et al. [2008]. They showed a secular density change of $-2.68 \pm 0.49 \%$ per decade at $400 \mathrm{~km}$, with trends of approximately -5 and $-2 \%$ per decade at solar minimum and maximum, respectively. Their previous study [Emmert et al., 2004] estimated a density trend of $-2 \%$ per decade at $200 \mathrm{~km}$ altitude, increasing to $-5 \%$ per decade at $700 \mathrm{~km}$. This earlier study agrees more closely with the results presented in our study, with the mean of our results showing a density trend of $-4 \%$ per decade at $200 \mathrm{~km}$, increasing to $-6 \%$ at $600 \mathrm{~km}$. However, the errors associated with the TLE ephemerides make a direct comparison difficult as the trend values contain large 


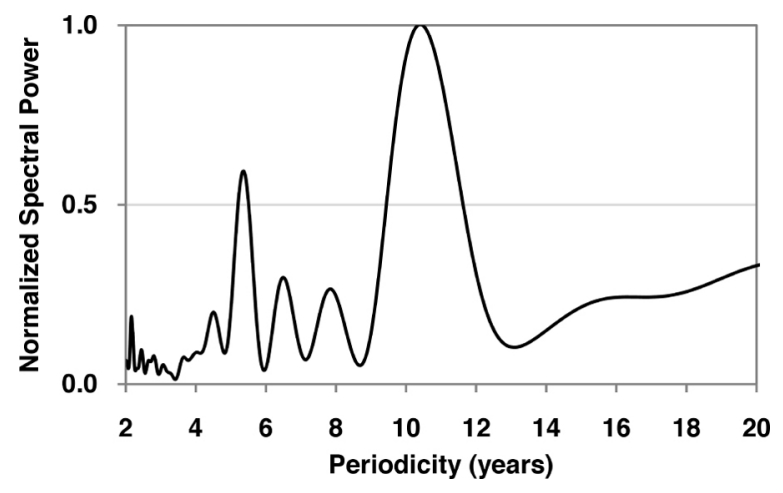

Figure 11. The combined spectral analysis data from the five altitude bands.

errors that increase with altitude. This makes the connection of similarity with the study by Emmert et al. tentative. Also, the studies by Marcos et al. [2005] and Qian et al. [2006] both obtained a secular density decrease at approximately $400 \mathrm{~km}$ of $1.7 \%$ per decade. This is approximately $3 \%$ more than our results. However, out of all the altitude bins included in our results, the region of $350-450 \mathrm{~km}$ shows the most variability from the linearly approximated altitude dependent trend. Qian et al. go on to estimate that if data were used up to the end of solar cycle $24(\sim 2008)$ then the secular trend would be greater at approximately $2.7 \%$. Therefore, agreement between our results and those of Marcos et al. and Qian et al. are both possible. Both our study and the study by Emmert et al. [2004] use much of the same satellite TLE data; and thus our investigation of the same phenomena using a different method has served to validate the existing estimates of the secular rate of thermospheric density decrease.

\subsection{Fourier Spectral Analysis}

[67] To investigate the nonlinear behavior and whether the rate of thermospheric density change could be connected with any particular natural phenomena, a Fourier spectral analysis was performed at five altitude locations: 380,400 , 430,560 and $580 \mathrm{~km}$. The bandwidth of periodicities analyzed ranged from 0 to $\sim 20$ years. Any periodicity longer than 20 years would not be clearly identifiable within a sample of 40 years, as in our study, so a 20 -year periodicity was deemed the upper boundary. Figure 10 shows the spectral analyses from the five altitude bands.

[68] All altitude bands show good agreement with the major peaks in periodicities. To refine the general results, the five spectral analyses were combined and normalized to highlight the most prominent periodicities. By doing this, altitude dependence was lost and so only general thermospheric behavior can be deduced. Figure 11 shows the combined spectral analyses. From this we can see two significant periodicities existing in the derived density ratios. Unsurprisingly, we can see the most powerful periodicity coinciding with the 11-year solar cycle at approximately 10.47 years. Therefore, realizing a connection of long-term secular density change with varying levels of solar flux merits the attention given earlier when attempting to quantify a relationship between these parameters.
[69] The second most powerful periodicity peaks at approximately 5.37 years. It shows a strong correlation with semi-solar cycle variation, suggesting a thermospheric reaction during times of transition from high to low solar activity. This is supported by the density ratios derived from Emmert et al. [2004] and Marcos et al. [2005] where they present two minima in density ratios: one at solar minimum and the other at solar maximum.

[70] The increase in spectral power for the periods of 13 years and longer is thought to be a manifestation of the secularly declining nature of the density ratios. It is possible that the Fourier spectral analysis confused the declining trend as an initial part of a waveform with longer periodicity than the historical 40-year period. Only further analysis beyond the scope of this study would provide a definitive answer.

[71] With regards to the other frequency peaks, their causes are less clear and would require a more detailed study to investigate further. However, we have shown that in our limited data set of 41 satellites there is a reasonable case for the existence of periodic variations with approximate lengths of $6.48,7.85$ years.

\section{Conclusions}

[72] We used a new method of estimating satellite ballistic coefficients as well as deriving changes in thermospheric density combined with the TLE data from 41 satellites over the historical period between the years 1970 and 2010 to show a long-term secular density trend existing in the thermosphere. On average, the secular density change is shown to decrease from approximately -4 to $-6 \%$ per decade from 200 to $600 \mathrm{~km}$ respectively. This agrees with the initial study by Emmert et al. [2004], who used much of the same satellite TLE data. From the differences seen between the first and second study by Emmert et al. [2004, 2008], it appears worthwhile to extend the range of this study to cover significantly more satellite TLE data. However, the results support the existing mode of thought regarding long-term global thermospheric density change.

[73] From a Fourier spectral analysis of the derived density data, four major periodicities were shown to exist over the 40-year historical period. Therefore, the long-term secular change has been validated as a nonlinear phenomenon. As previously mentioned a more detailed study using more satellite data would give a better picture of periodicities in thermospheric density change. We showed strong evidence of a relationship between thermospheric density change and solar activity validating our analysis of the derived density data during times of high and low solar flux. It has been shown that thermospheric cooling increases, relatively, during times of low activity. Only relative changes of cooling rates between high and low levels of solar flux can be inferred owing to the particular method used in this study.

[74] With the increasing amount of evidence for a longterm thermospheric density reduction, any system affected by atmospheric density in this region needs to be reevaluated. The most significant effects would be to orbiting objects, as a reduction in thermospheric density would remove the natural sink of orbit degradation; good news for propellant management systems on board most 
orbit-maintained satellites; bad news for the space debris population and increasing risks of catastrophic collisions.

[75] Acknowledgments. Robert Lysak thanks the reviewers for their assistance in evaluating this paper.

\section{References}

Aksnes, K. (1974), Short-periodic and long-periodic perturbations of a spherical satellite due to direct solar radiation, Preprint Ser. 230, Cent. for Astrophys., Cambridge, Mass.

Analytical Graphics, Inc. (2009), Satellite tool kit, Exton, Pa. [Available at http://www.agi.com.]

Emmert, J. T., J. M. Picone, J. L. Lean, and S. H. Knowles (2004), Global change in the thermosphere: Compelling evidence of a secular decrease in density, J. Geophys. Res., 109, A02301, doi:10.1029/2003JA010176.

Emmert, J. T. J. M. Picone, J. L. Lean, and R. R. Meier (2008), Thermospheric global average trends, 1967-2007, derived from orbits of 5000 near-Earth objects, Geophys. Res. Lett., 35, L05101, doi:10.1029/ 2007GL032809.

Emmert, J. T., J. L. Lean, and J. M. Picone (2010), Record-low thermospheric density during the 2008 solar minimum, Geophys. Res. Lett., 37, L12102, doi:10.1029/2010GL043671.

Erwin, E. H. (2007), NOAA Indices, ftp://ftp.ngdc.noaa.gov/, Natl. Geophys. Data Cent., Boulder, Colo.

Hoots, F. R., and R. L. Roehrhich (1980), Models for propagation of NORAD element sets, Spacetrack Rep. 3, 87 pp., Off. of Astrodyn. Aerosp. Def. Cent., Peterson Air Force Base, Colo.

Keating, G. M., R. H. Tolson, and M. S. Bradford (2000), Evidence of longterm global decline in the Earth's thermospheric densities apparently related to anthropogenic effects, Geophys. Res. Lett., 27, 1523-1526, doi:10.1029/2000GL003771.

King-Hele, D. (1987), Satellite Orbits in an Atmosphere: Theory and Applications, Blackie Acad. and Prof., Glasgow, U. K.

Liu, L., W. Wan, B. Ning, O. M. Pirog, and V. I. Kurkin (2006), Solar activity variations of the ionospheric peak electron density, J. Geophys. Res., 111, A08304, doi:10.1029/2006JA011598.

Marcos, F. A., J. O. Wise, M. J. Kendra, N. J. Grossbard, and B. R. Bowman (2005), Detection of a long-term decrease in thermospheric neutral density, Geophys. Res. Lett., 32, L04103, doi:10.1029/2004GL021269.

McCarthy, D. D. (1996), IERS conventions, Tech. Note 21, Cent. Bur. of IERS, Obs. de Paris, Paris.
Moe, K., and B. R. Bowman (2005), The effects of surface composition and treatment on drag coefficients of spherical satellites, paper presented at Astrodynamics Specialist Conference, Am. Inst. of Aeronaut. and Astronaut., Lake Tahoe, Calif.

Montenbruck, O., and E. Gill (2005), Satellite Orbits: Models, Methods, Applications, Springer, Berlin.

Picone, J. M., A. E. Hedin, D. P. Drob, and A. C. Aikin (2002), NRLMSISE-00 empirical model of the atmosphere: Statistical comparisons and scientific issues, J. Geophys. Res., 107(A12), 1468, doi:10.1029/2002JA009430.

Picone, J. M., J. T. Emmert, and J. L. Lean (2005), Thermospheric densities derived from spacecraft orbits: Accurate processing of two-line element sets, J. Geophys. Res., 110, A03301, doi:10.1029/2004JA010585.

Qian, L. R., G. Roble, S. C. Solomon, and T. J. Kane (2006), Calculated and observed climate change in the thermosphere, and a prediction for solar cycle 24, Geophys. Res. Lett., 33, L23705, doi:10.1029 2006GL027185.

Roble, R. G., and R. E. Dickinson (1989), How will changes in carbon dioxide and methane modify the mean structure of the mesosphere and thermosphere?, J. Geophys. Res., 16, 1441-1444.

Roble, R. G., and D. S. Ramesh (2002), Cooling mechanisms of the planetary thermospheres: The key role of $\mathrm{O}$ atom vibrational excitation of $\mathrm{CO} 2$ and NO, ChemPhysChem, 3, 841-843.

Saunders, A., H. G. Lewis, and G. G. Swinerd (2009), A new tool for satellite re-entry predictions, paper presented at 5th European Conference of Space Debris, Eur. Space Agency, Darmstadt, Germany, March-April.

Tapley, B. D., et al. (1996), The Joint Gravity Model 3, J. Geophys. Res., 101, 28,029-28,049, doi:10.1029/96JB01645.

Tobiska, K. W., and C. A. Barth (1990), A solar EUV flux model, J. Geophys. Res., 95, 8243-8251, doi:10.1029/JA095iA06p08243.

Vallado, D. A. (1997), Fundamentals of Astrodynamics and Applications, Kluwer Acad., Dordrecht, Netherlands.

van der Ha, J. C., and V. J. Modi (1977), Analytical evaluation of solar radiation induced perturbations of space structures, J. Astronaut. Sci., 25(4), 283-306.

Yeomans, D. K. (2009), NASA Horizons system, Natl. Aeronaut. and Space Admin., Washington, D. C. [Available at http://ssd.jpl.nasa.gov/ ?horizons.]

H. Lewis, A. Saunders, and G. Swinerd, Astronautics Research Group, School of Engineering Sciences, University of Southampton, Highfield SO171BJ, UK. (hglewis@soton.ac.uk; a.saunders@soton.ac.uk; g.g. swinerd@soton.ac.uk) 